\title{
Integral order RF and microwave photonic based signal processors using Kerr soliton crystal micro- combs
}

\author{
David J. Moss \\ Optical Sciences Centre, Swinburne University of Technology, Hawthorn, VIC 3122, Australia.

\begin{abstract}
Soliton crystal micro-combs are powerful tools as sources of multiple wavelength channels for radio frequency $(\mathrm{RF})$ signal processing. They offer a compact device footprint, large numbers of wavelengths, very high versatility, and wide Nyquist bandwidths. Here, we demonstrate integral order RF signal processing functions based on a soliton crystal micro-comb, including a Hilbert transformer and first- to third-order differentiators. We compare and contrast results achieved and the tradeoffs involved with varying comb spacing, tap design methods, as well as shaping methods.
\end{abstract}

Keywords: RF photonics, Optical resonators

\section{Introduction}

RF signal processing functions, including the Hilbert transform and differentiation, are building blocks of advanced RF applications such as radar systems, single sideband modulators, measurement systems, speech processing, signal sampling, and communications [1-42]. Although the electronic digital-domain tools that are widely employed enable versatile and flexible signal processing functions, they are subject to the bandwidth bottleneck of analog-to-digital convertors [4], and thus face challenges in processing wideband signals.

Photonic RF techniques [1-3] have attracted great interest during the past two decades with their capability of providing ultra-high bandwidths, low transmission loss, and strong immunity to electromagnetic interference. Many approaches to photonic RF signal processing have been proposed that take advantage of the coherence of the RF imprinted optical signals - thereby inducing optical interference. These coherent approaches map the response of optical filters, implemented through optical resonators or nonlinear effects, onto the RF domain [7-12]. As such, the ultimate performance of the RF filters largely depends on the optical filters. State-of-art demonstrations of coherent photonic RF filters include those that use integrated micro-ring resonators, with $\mathrm{Q}$ factors of > 1 million, as well as techniques that employ on-chip (waveguide-based) stimulated Brillouin scattering [10-12].
Both of these approaches have their unique advantages - the former uses passive devices and so can achieve very low power consumption, while Brillouin scattering can achieve a much higher frequency selectivity, reaching a $3 \mathrm{~dB}$ bandwidth resolution as low as $32 \mathrm{MHz}$.

Coherent approaches generally focus on narrow-band applications where the frequency range of concern is narrow and the focus is on frequency selectivity, and where the filters are generally band-pass or band-stop in nature. In contrast, incoherent approaches that employ transversal filtering structures can achieve a very diverse range of functions with a much wider frequency range, such as Hilbert transforms and differentiators. The transversal structure originates from the classic digital finite impulse response filter, where the transfer function is achieved by weighting, delaying and summing the input signals. Unlike digital approaches that operate under von-Neumann protocols, photonic implementations achieve the entire process through analog photonics, where the weighting, delaying and summing happens physically at the location of the signals, instead of reading and writing backand-forth from memory.

To achieve the transversal structure optically, four steps are required. First, the input RF signals are replicated, or multicast, onto multiple wavelengths simultaneously using wavelengths supplied from either multiple single wavelength, or single multiple wavelength, sources. Next, the replicated signals are assigned different weights for each wavelength and then the composite signal is progressively delayed where each 


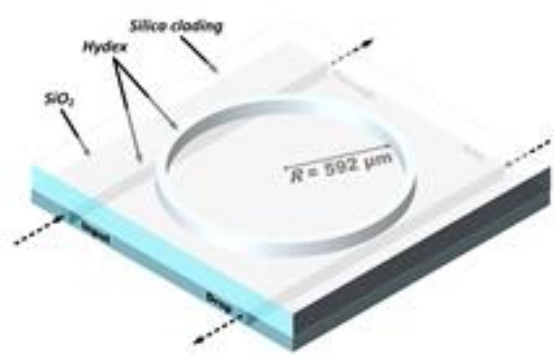

(a)

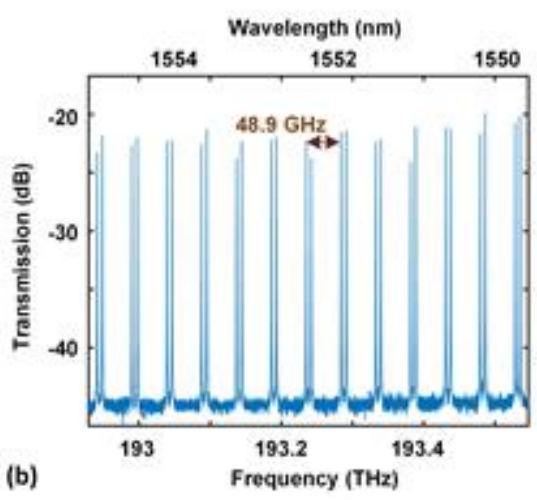

(b)

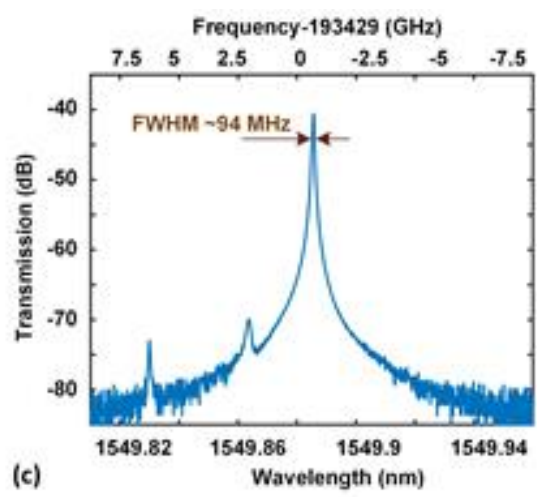

Fig. 1. (a) Schematic of the micro-ring resonator. (b) Drop-port transmission spectrum of the integrated MRR with a span of 5 nm, showing an optical free spectral range of $48.9 \mathrm{GHz}$. (c) A resonance at $193.429 \mathrm{THz}$ with a full width at half maximum (FWHM) of $\sim 94 \mathrm{MHz}$, corresponding to a quality factor of $\sim 2 \times 10^{6}$.

wavelength is incrementally delayed relative to the next. Finally, the weighted replicas are summed together by photodetecting the entire signal. The underpinning principle to this process is to physically achieve multiple parallel channels where each channel carries and processes one replica of the RF signal. In addition to wavelength multiplexing techniques, this can also be accomplished with spatial multiplexing, such using an array of fibre delay lines to spatially achieve the required parallelism. Although this is straightforward to implement, it suffers from severe tradeoffs between the number of channels and overall footprint and cost. Exploiting the wavelength dimension is a much more elegant approach since it makes much better use of the wide optical bandwidth of over the $10 \mathrm{THz}$ that the telecommunications $\mathrm{C}$ band offers, and thus is more compact. However, traditional approaches to generating multiple optical wavelengths have been based on discrete laser arrays, [6-9] and these face limitations in terms of a large footprint, relatively high cost, and challenges in terms of accurate control of the wavelength spacing.

Optical frequency combs - equally spaced optical frequency lines - are a powerful approach to implementing incoherent photonic RF filters since they can provide a large number of wavelength channels with equal frequency spacings, and in a compact scheme. Among the many traditional methods of achieving optical frequency combs, electro-optic (EO) techniques have probably experienced the widest use for RF photonics. By simultaneously driving cascaded EO modulators with a high-frequency RF source, a large number of comb lines can be generated, and these have been the basis of many powerful functions. However, EO combs are not without challenges. On the one hand, they generally have a small Nyquist zone (half of the frequency spacing), limited by the RF source. On the other hand, the employed bulky optical and RF devices are challenging to be monolithically integrated. As such, to overcome the hurdles of size, reliability and cost-effectiveness of bulky photonic RF systems, integrated frequency combs would represent a highly attractive approach.

Integrated Kerr optical frequency combs [47-76], or microcombs, that originate via optical parametric oscillation in monolithic micro-ring resonators (MRRs), have recently come into focus as a fundamentally new and powerful tool due to their ability to provide highly coherent multiple wavelength channels in integrated form, from a single source. They offer a much higher number of wavelengths than typically is available through EO combs, together with a wide range of comb spacings (free spectral range (FSR)) including ultralarge FSRs, as well as greatly reduced footprint and complexity. Micro-combs have enabled many fundamental breakthroughs [50] including ultrahigh capacity communications [77-79], neural networks [80-82], complex quantum state generation [83-97] and much more. In particular, micro-combs have proven to be very powerful tools for a wide range of RF applications such as optical true time delays [31], transversal filters [34, 38], signal processors [29, 32], channelizers [37] and others [15, 18, 26-28, 36, 39-41]. They have greatly enhanced the performance of RF signal processors in terms of the resolution (for coherent systems) and operation bandwidth (for incoherent systems).

In one of the first reports of using micro-combs for RF signal processing, we demonstrated a Hilbert transformer based on a transversal filter that employed up to 20 taps, or wavelengths. [36] This was based on a $200 \mathrm{GHz}$ FSR spaced micro-comb source that operated in a semi-coherent mode that did not feature solitons. Nonetheless, this provided a low enough noise comb source to enable very attractive performance, achieving a bandwidth of over 5 octaves in the RF domain. Subsequently, [15] we demonstrated $1^{\text {st }} 2^{\text {nd }}$ and $3^{\text {rd }}$ order integral differentiators based on the same $200 \mathrm{GHz}$ source, achieving high RF performance with bandwidths of over $26 \mathrm{GHz}$, as well as a range of RF spectral filters including bandpass, tunable bandpass and gain equalizing filters [32, 33]. 
Recently, a powerful category of micro-combs - soliton crystals - has been reported [59, 76, 98]. It features ultra-low intensity noise states and straightforward generation methods via adiabatic pump wavelength sweeping. Soliton crystals are unique solutions to the parametric dynamics governed by the Lugiato-Lefever equation. They are tightly packaged solitons circulating along the ring cavity, stabilized by a background wave generated by a mode-crossing. Due to their much higher intra-cavity intensity compared with the single-soliton states of DKS solitons, thermal effects that typically occur during the transition from chaotic to coherent soliton states are negligible, thus alleviating the need for complex pump sweeping methods.

We have exploited soliton crystal states generated in record low FSR (49 GHz) micro-ring resonators (MRRs), thus generating a record large number of wavelengths, or taps, to achieve a wide range of high performance RF signal processing functions. These include RF filters [35], true time delays [30], RF integration [42], fractional Hilbert transforms [27], fractional differentiation [41], phase-encoded signal generation [26], arbitrary waveform generation [43], filters realized by bandwidth scaling [38], and RF channelizers [44] and much more including quantum optical devices [99-174].

In this work, we further examine transversal photonic RF signal processors that exploit soliton crystal micro-combs. We demonstrate Hilbert transformers as well as $1^{\text {st }}, 2^{\text {nd }}$, and $3^{\text {rd }}$ order integral differentiators and explore in detail the tradeoffs inherent between using differently spaced soliton crystal micro-combs as well as different numbers of tap weights and design methods. Our study sheds light on the optimum number of taps, while the experimental results agree well with theory, verifying the feasibility of our approach towards the realization of high-performance photonic RF signal processing with potentially reduced cost, footprint and complexity.

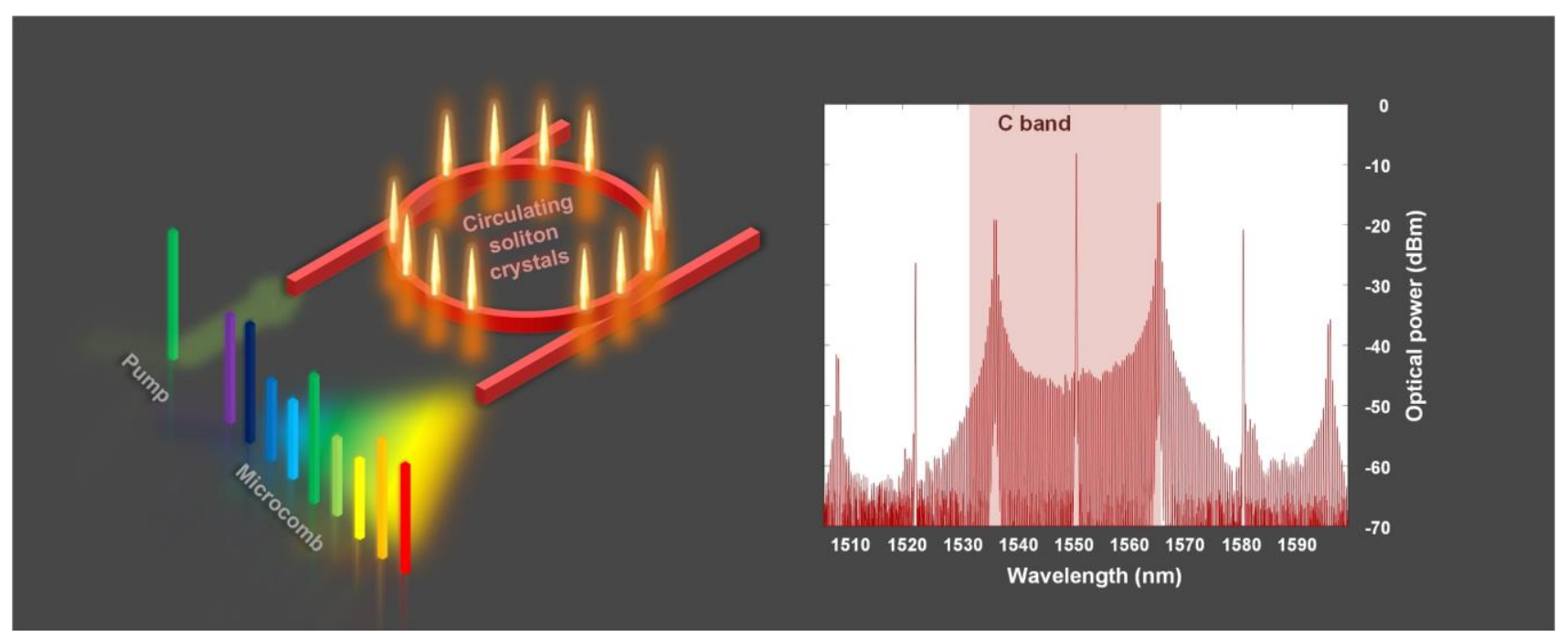

Fig. 2. Schematic illustration of the integrated MRR for generating the Kerr frequency comb and the optical spectrum of the generated soliton crystal combs with a $100-n m$ span. 

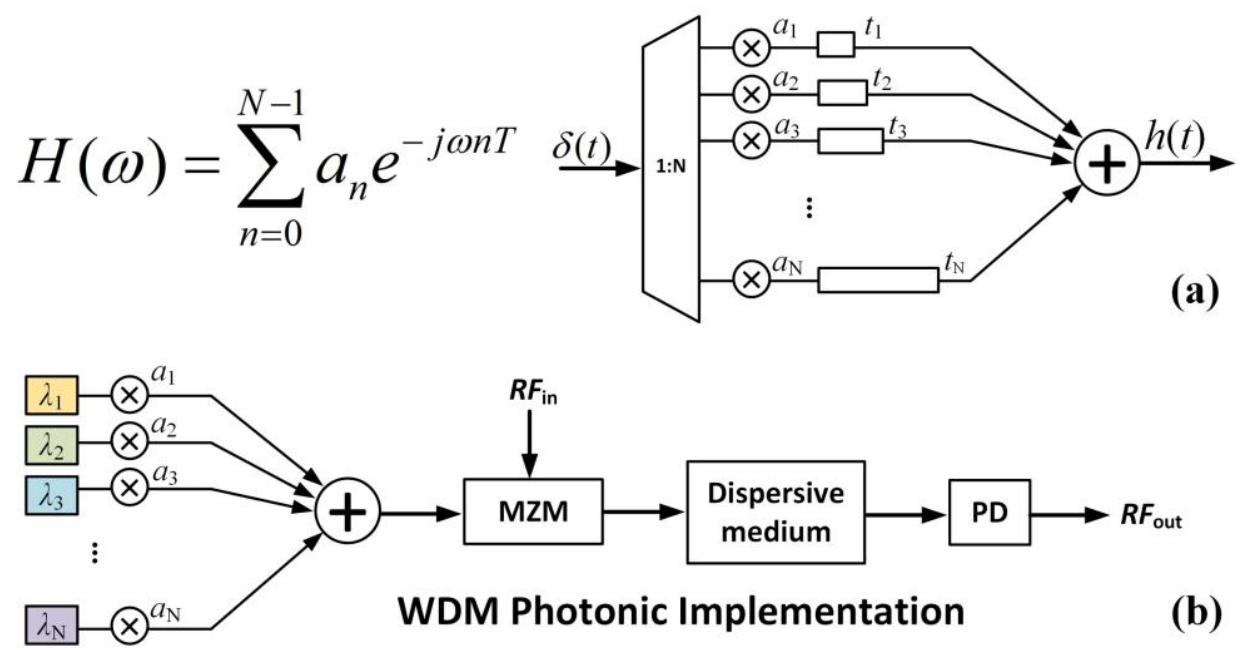

(b)

Fig. 3. Conceptual diagram of the transversal structure.

\section{Operation principle}

The generation of micro-combs is a complex process that generally relies on a high nonlinear material refractive index, low linear and nonlinear loss, as well as engineered anomalous dispersion [59-64]. Diverse platforms have been developed for micro-comb generation [58], such as silica, magnesium fluoride, silicon nitride, and doped silica glass. The MRR used to generate soliton crystal micro-combs is shown in Fig. 1 (a). It was fabricated on a high-index doped silica glass platform using CMOS compatible processes. Due to the ultra-low loss of our platform, the MRR features narrow resonance linewidths, corresponding to quality factors as high as 1.5 million, with radii of $\sim 592 \mu \mathrm{m}$, which corresponds to a very low FSR of $\sim 0.393 \mathrm{~nm}(\sim 48.9 \mathrm{GHz})$ (Fig. 1 (b)) [54-55, 3940]. First, high-index $(n=\sim 1.7$ at $1550 \mathrm{~nm})$ doped silica glass films were deposited using plasma-enhanced chemical vapour deposition, followed by patterning with deep ultraviolet stepper mask photolithography and then etched via reactive ion etching followed by deposition of the upper cladding. The device architecture typically uses a vertical coupling scheme where the gap (approximately $200 \mathrm{~nm}$ ) can be controlled via film growth - a more accurate approach than lithographic techniques. The advantages of our platform for optical microcomb generation include ultra-low linear loss $\left(\sim 0.06 \mathrm{~dB} \cdot \mathrm{cm}^{-}\right.$ $\left.{ }^{1}\right)$, a moderate nonlinear parameter $\left(\sim 233 \mathrm{~W}^{-1} \cdot \mathrm{km}^{-1}\right)$ and, in particular, a negligible nonlinear loss up to extremely high intensities $\left(\sim 25 \mathrm{GW} \cdot \mathrm{cm}^{-2}\right)$ [65-76]. After packaging the device with fibre pigtails, the through-port insertion loss was as low as $0.5 \mathrm{~dB} /$ facet, assisted by on-chip mode converters.

To generate soliton crystal micro-combs, we amplified the pump power up to $30.5 \mathrm{dBm}$. When the detuning between the pump wavelength and the cold resonance became small enough, such that the intra-cavity power reached a threshold value, modulation instability (MI) driven oscillation was initiated. Primary combs were thus generated with a spacing determined by the MI gain peak - mainly a function of the intra-cavity power and dispersion. As the detuning was changed further, distinctive 'fingerprint' optical spectra were observed (Fig. 2), similar to what has been reported from spectral interference between tightly packed solitons in a cavity - so-called 'soliton crystals' [55-56]. The second power

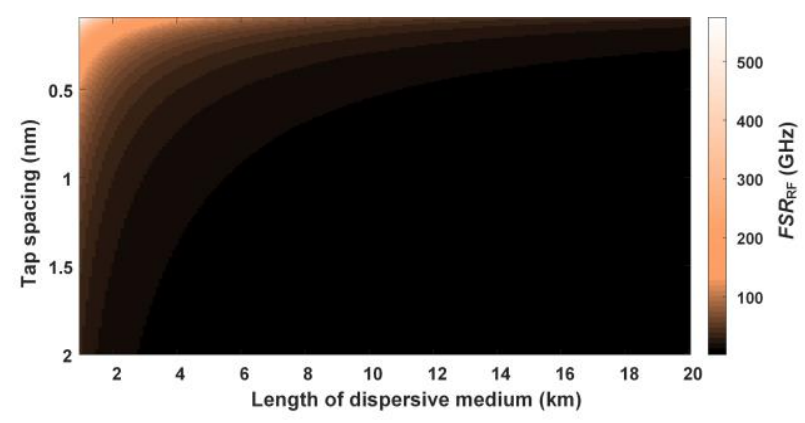

Fig. 4. Free spectral range of the RF transversal signal processor according to the length of fibre and comb spacing. Here we used single mode fibre with the second order dispersion coefficient of $\beta=\sim 17.4$ $\mathrm{ps} / \mathrm{nm} / \mathrm{km}$ at $1550 \mathrm{~nm}$ for the calculation of $F S R_{\mathrm{RF}}$.

step jump in the measured intra-cavity power was observed at this point, where the soliton crystal spectra appeared. We found that it was not necessary to achieve any specific state, including either soliton crystals or single soliton states, in order to obtain high performance - only that the chaotic regime [59] should be avoided. Nonetheless, the soliton crystals states provided the lowest noise states of all our micro-combs and have also been used as the basis for a microwave oscillator with low phase-noise [28]. This is important since there is a much wider range of coherent low RF noise states that are more readily accessible than any specific soliton related state [59]. 


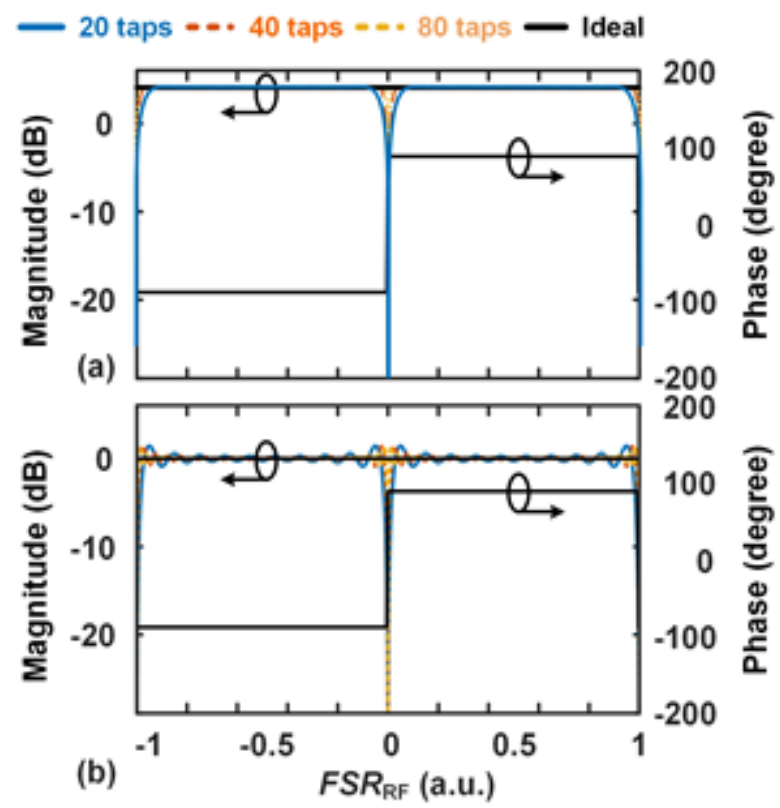

Fig. 5. Theoretical and simulated RF magnitude according to the number of taps and ideal phase response of Hilbert transformer with $90^{\circ}$ phase shift. (a) With a hamming window applied. (b) Without window method applied.

Figure 3 illustrates the conceptual diagram of the transversal structure. A finite set of delayed and weighted replicas of the input RF signal are produced in the optical domain and then combined upon detection. The transfer function of a general transversal signal processor can be described as

$$
H(\omega)=\sum_{n=0}^{N-1} a_{n} e^{-j \omega n T}
$$

where $N$ is the number of taps, $\omega$ is the RF angular frequency, $T$ is the time delay between adjacent taps, and $a_{\mathrm{n}}$ is the tap coefficient of the $n_{\text {th }}$ tap, which is the discrete impulse response of the transfer function $F(\omega)$ of the signal processor. The discrete impulse response $a_{\mathrm{n}}$ can be calculated by performing the inverse Fourier transform of the transfer function $F(\omega)$ of the signal processor [11]. The free spectral range of the RF signal processor is determined by $T$, since $F S R_{\mathrm{RF}}=1 / T$. As the multi-wavelength optical comb is transmitted through the dispersive medium, the time delay can be expressed as

$$
T=D \times L \times \Delta \lambda
$$

where $D$ denotes the dispersion coefficient, $L$ denotes the length of the dispersive medium, and $\Delta \lambda$ represents the wavelength spacing of the soliton crystal micro-comb, as shown in Fig. 4, which indicates the potentially broad bandwidth RF signal that the system can process. From Figure 4 we can see the relationship between the wavelength spacing of the comb, the total delay of the fibre, and the resulting RF FSR, or essentially Nyquist zone. The operation bandwidth

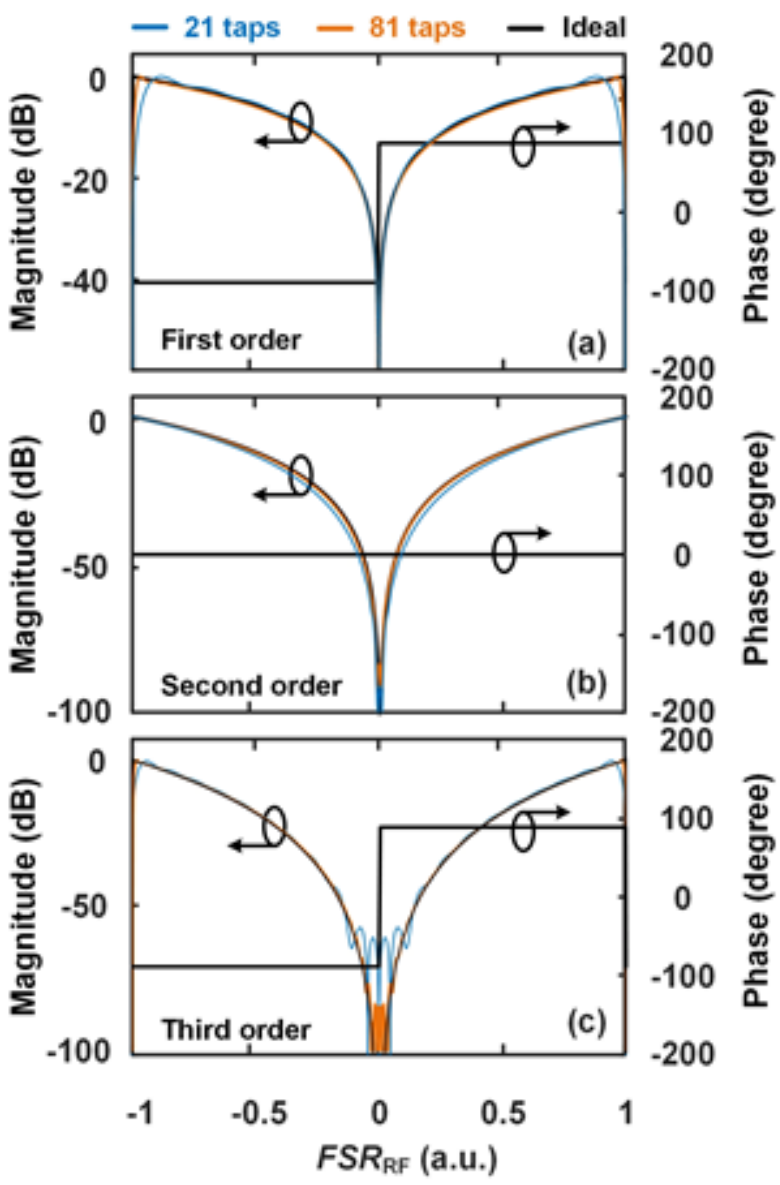

Fig. 6. Theoretical and simulated RF magnitude according to the number of taps and ideal phase response of (a) first-order differentiator. (b) second-order differentiator. (c) third-order differentiator.

can be easily adjusted by changing the time delay (i.e., using different delay elements). The maximum operational bandwidth of the transversal signal processor is limited by the comb spacing (i.e., the Nyquist frequency, or half of the comb spacing). Thus, employing a comb shaping method to achieve a larger comb spacing could enlarge the maximum operational bandwidth, although at the expense of providing fewer comb lines/taps across the C-band. Hence, the number of comb lines/taps as well as the comb spacing, are key parameters that determine the performance of the signal processor. We investigate this tradeoff in detail in this paper.

Figures 5 and 6 show the theoretically calculated performance of the Hilbert transformer with a $90^{\circ}$ phase shift together with the $1^{\text {st }}, 2^{\text {nd }}$ and $3^{\text {rd }}$ order integral differentiators in terms of their filter amplitude response, as a function of the number of taps. Note that a Hamming window [11] is applied in Fig. 5 (a) in order to suppress the sidelobes of the Hilbert transformer. As seen in Fig. 7, the theoretical $3 \mathrm{~dB}$ bandwidth increases rapidly with the number of taps. 


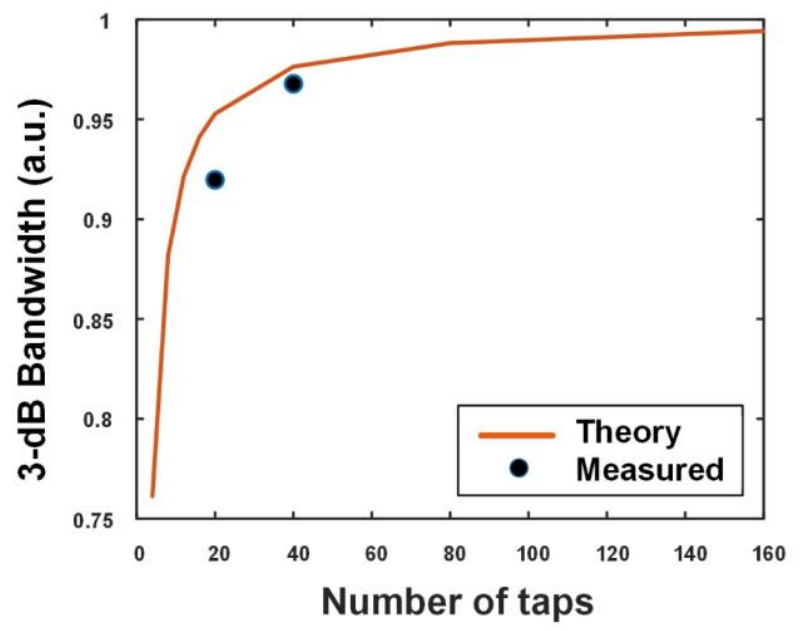

Fig. 7. Simulated and experimental results of $3-\mathrm{dB}$ bandwidth with different taps for a Hilbert transformer with $90^{\circ}$ phase shift.

\section{Experiment}

Figure 8 shows the experimental setup of the transversal filter signal processor based on a soliton crystal micro-comb. It consists mainly of two parts - comb generation and flattening followed by the transversal structure. In the first part, the generated soliton crystal micro-comb was spectrally shaped with two WaveShapers to enable a better signal-tonoise ratio as well as a higher shaping accuracy. The first WaveShaper (WS1) was used to pre-flatten the scallop-shaped comb spectrum that is a hallmark of soliton crystal microcombs. In the second part, the flattened comb lines were modulated by the RF input signal, effectively multicasting the $\mathrm{RF}$ signal onto all of the wavelength channels to yield replicas. The RF replicas were then transmitted through a

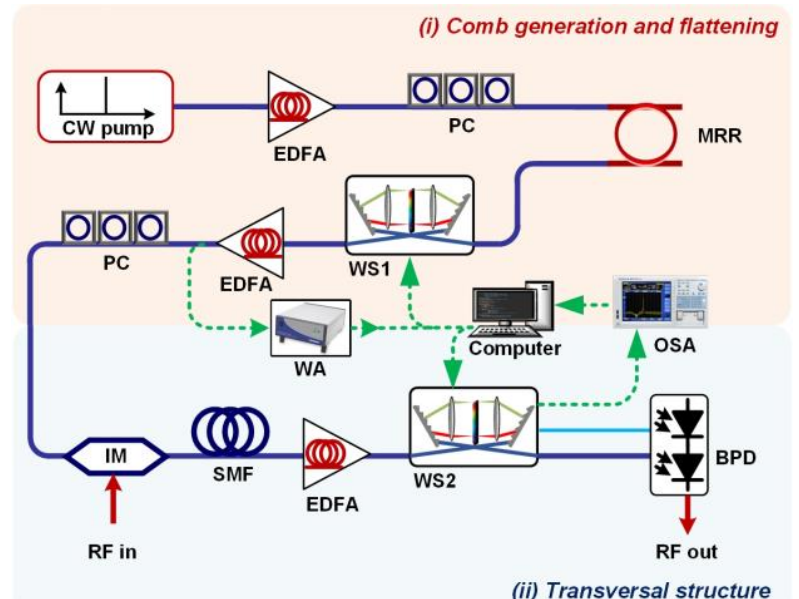

Fig. 8. Experimental set up of RF signal processor based on soliton crystal micro-comb source. CW: Continuously wave. EDFA: Erbiumdoped fibre amplifier. PC: Polarization controller. WS: WaveShaper. IM: Intensity modulator. SMF: Single mode fibre. BPD: Balanced photodetector. WA: wave analyzer. OSA: optical spectral analyzer spool of standard SMF $(\beta=\sim 17.4 \mathrm{ps} / \mathrm{nm} / \mathrm{km}$ ) to obtain a progressive time delay between the adjacent wavelengths. Next, the second WaveShaper (WS2) equalized and weighted the power of the comb lines according to the designed tap coefficients. To increase the accuracy, we adopted a real-time feedback control path to read and shape the power of the comb lines accurately. Finally, the weighted and delayed taps were combined and converted back into the RF domain via a highspeed balanced photodetector (Finisar, $43 \mathrm{GHz}$ bandwidth).

Figure 9 shows the experimental results for the Hilbert transformer with a $90^{\circ}$ phase shift. The shaped optical combs are shown in Figs. 9 (a) (e) (i). A good match between the measured comb lines' power (blue lines for positive, black lines for negative taps) and the calculated ideal tap weights (red dots) was obtained, indicating that the comb lines were successfully shaped. Note that we applied a Hamming window [11] for single-FSR (49 GHz) and 4-FSR (196 GHz) comb spacings when designing the tap coefficients. One can see that with a Hamming window applied, the deviation of the amplitude response from the theoretical results can be improved. Figs. 9 (b) (f) (j) show the measured and simulated amplitude response of the Hilbert transformer using singleFSR, 2-FSR, and 4-FSR comb spacings, respectively. The corresponding phase responses are depicted in Figs. 9 (c) (g) (k). It can be seen that all three configurations exhibit the response expected from the ideal Hilbert transform. The system demonstration for the Hilbert transform with real-time signals consisting of a Gaussian input pulse, generated by an arbitrary waveform generator (AWG, KEYSIGHT M9505A) was also performed, as shown in Figs. 9 (d) (h) (l) (black solid curves). They were recorded by means of a high-speed realtime oscilloscope (KEYSIGHT DSOZ504A Infinium). For comparison, we also depict the ideal Hilbert transform results, as shown in Figs. 9 (d) (h) (l) (blue dashed curves). For the Hilbert transformer with single-FSR, 2-FSR, and 4-FSR comb spacings, the calculated RMSEs between the measured and the ideal curves are $\sim 0.133, \sim 0.1065$, and $\sim 0.0957$, respectively. The detailed performance parameters are listed in Table 1.

Figure 10 shows the experimental results for the differentiators with increasing integral orders of 1,2 , and 3 . The shaped optical spectra in Figs. 10 (a) (e) (i) (m) (q) (u) show a good match between the measured comb lines' power and the calculated ideal tap weights. Figures. 10 (b) (f) (j) (n) (r) (v) show measured and simulated amplitude responses of the differentiators. The corresponding phase response is depicted in Fig. 10 (c) (g) (k) (o) (s) (w) where it can be seen that all integral differentiators agree well with theory. Here, we use the WaveShaper to programmably shape the combs to simulate MMRs with different FSRs. By essentially artificially adjusting the comb spacing, we effectively obtain 

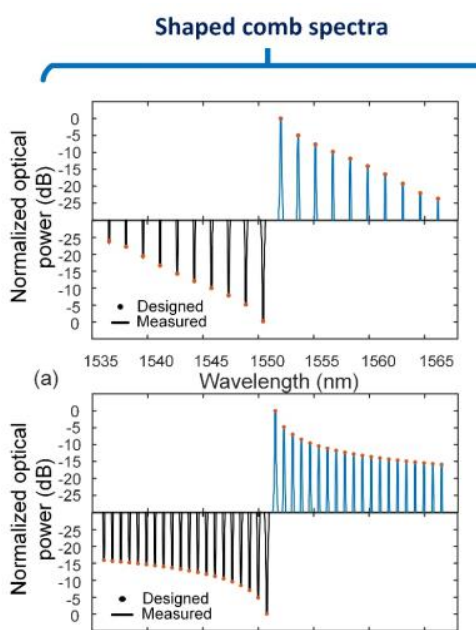

(e)
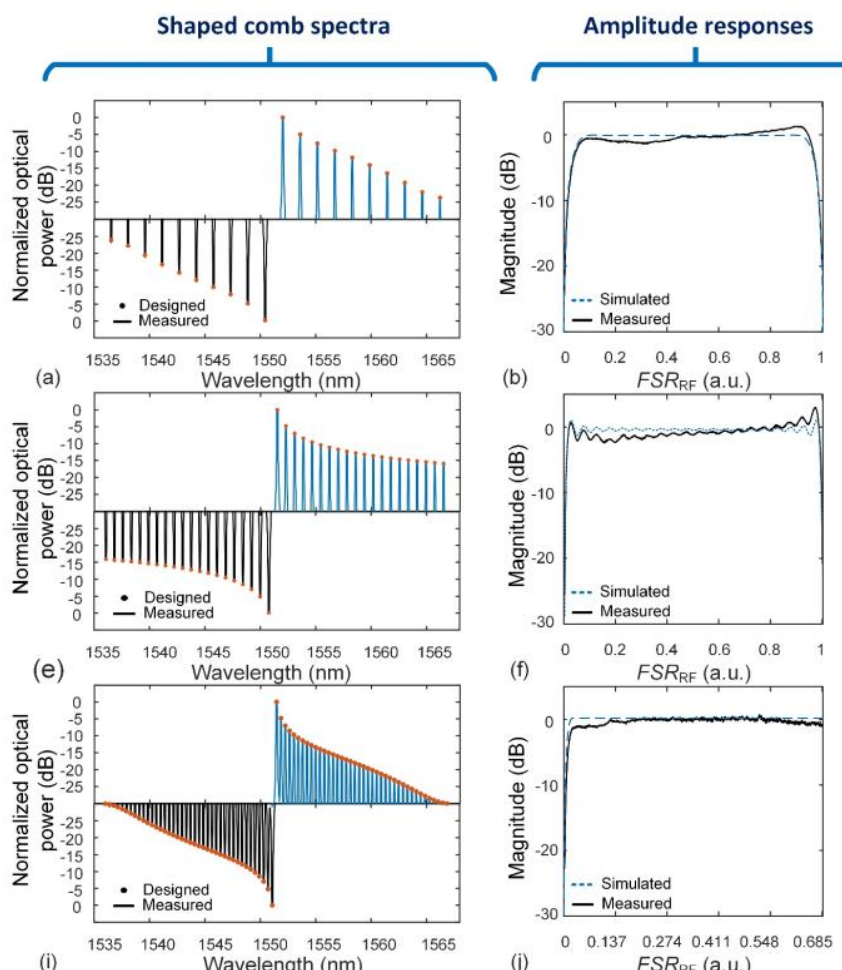

(j)
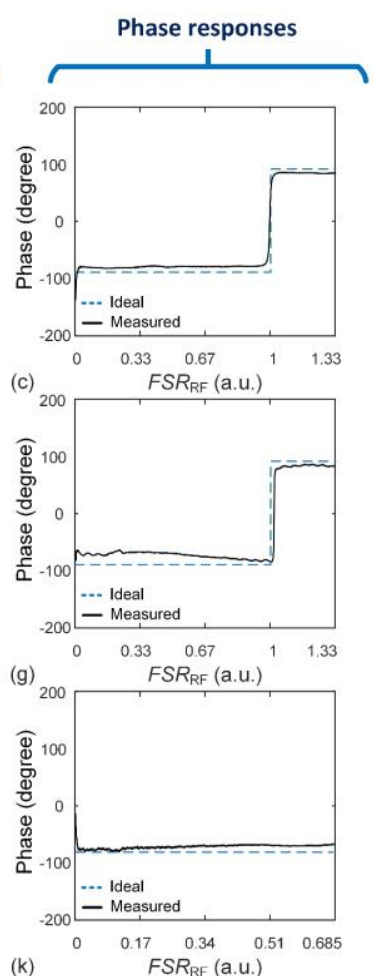

(k) $\quad \operatorname{FSR}_{\mathrm{RF}}$ (a.u.)
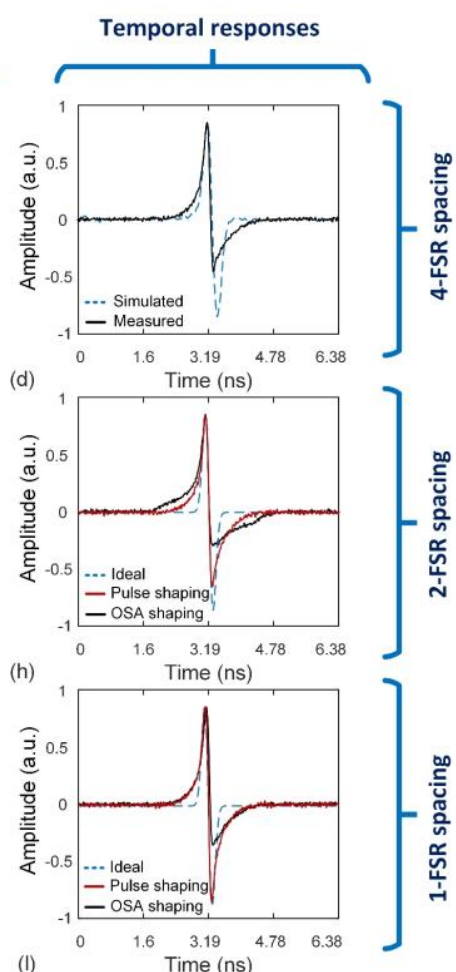

Fig. 9. Simulated and measured $90^{\circ}$ Hilbert transformer with varying comb spacing. (a) (e) (i) Shaped optical spectral. (b) (f) (j) Amplitude responses (the $|\mathrm{S} 21|$ responses measured by a Vector Network Analyzer). (c) (g) (k) Phase responses. (d) (h) (l) Temporal responses measured with a Gaussian pulse invut.

a variable operation bandwidth for the differentiator, which is advantageous for diverse requirements of different applications. Here, we normalised the FSR of the RF response to have the unique operational bandwidth for comparing the perfoamance of different processing functions in the same scales. For the $1^{\text {st }}, 2^{\text {nd }}$, and $3^{\text {rd }}$ order differentiators with a single-FSR (49 GHz) spacing, the calculated RMSEs between the measured and ideal curves are $\sim 0.1111, \sim 0.1139, \sim 0.1590$, respectively. For the $1^{\text {st }}, 2^{\text {nd }}$, and $3^{\text {rd }}$ order differentiators with a 4-FSR (196 GHz) spacing, the calculated RMSEs between the measured and ideal curves are $\sim 0.0838, \sim 0.0570, \sim 0.1718$, respectively. Note that there is some observed imbalance in the time-domain between the positive and negative response

to the Gaussian input pulse. This is due to the imbalance of the two ports of the balanced photodetector.

In order to reduce the errors mentioned above, for both the Hilbert transformer and the differentiator, we developed a more accurate comb shaping approach, where the error signal of the feedback loop was generated directly by the measured impulse response, instead of the optical power of the comb lines. We then performed the Hilbert transform and differentiation with the same transversal structure as the previous measurements, the results of which are shown in Figs. 9 (h) (I) and Fig. 10 (t). One can see that the imbalance of the response in time domain has been compensated, and the

TABLE I

PERFORMANCE OF OUR TRANSVERSAL SIGNAL PROCESSORS

\begin{tabular}{|c|c|c|c|c|c|c|c|}
\hline \multirow{2}{*}{ Type } & \multirow{2}{*}{$\begin{array}{l}\text { Number } \\
\text { of taps }\end{array}$} & \multirow{2}{*}{$\begin{array}{l}\text { Wavelength } \\
\text { spacing }\end{array}$} & \multirow{2}{*}{$\begin{array}{c}\text { Frequency } \\
\text { spacing }(\mathrm{GHz})\end{array}$} & \multirow{2}{*}{$\begin{array}{l}\text { Nyquist zone } \\
\quad(\mathrm{GHz})\end{array}$} & \multirow{2}{*}{ Octave } & \multicolumn{2}{|c|}{ Temporal pulse RMSE } \\
\hline & & & & & & OSA shaping & Pulse shaping \\
\hline Hilbert transformer & 20 & 4-FSR & 196 & 98 & $>4.5$ & $\sim 0.0957$ & 1 \\
\hline Hilbert transformer & 40 & 2-FSR & 98 & 49 & $>6$ & $\sim 0.1065$ & $\sim 0.0845$ \\
\hline Hilbert transformer & 80 & Single-FSR & 49 & 24.5 & 1 & $\sim 0.1330$ & $\sim 0.0782$ \\
\hline Differentiator $-1^{\text {st }}$ order & 21 & 4-FSR & 196 & 98 & I & $\sim 0.0838$ & 1 \\
\hline Differentiator $-2^{\text {nd }}$ order & 21 & 4-FSR & 196 & 98 & I & $\sim 0.0570$ & I \\
\hline Differentiator $-3^{\text {rd }}$ order & 21 & 4-FSR & 196 & 98 & I & $\sim 0.1718$ & I \\
\hline Differentiator $-1^{\text {st }}$ order & 81 & Single-FSR & 49 & 24.5 & I & $\sim 0.1111$ & 1 \\
\hline Differentiator $-2^{\text {nd }}$ order & 81 & Single-FSR & 49 & 24.5 & I & $\sim 0.1139$ & $\sim 0.0620$ \\
\hline Differentiator $-3^{\text {rd }}$ order & 81 & Single-FSR & 49 & 24.5 & I & $\begin{array}{l}\sim 1590 \\
\sim 0.150\end{array}$ & I \\
\hline
\end{tabular}



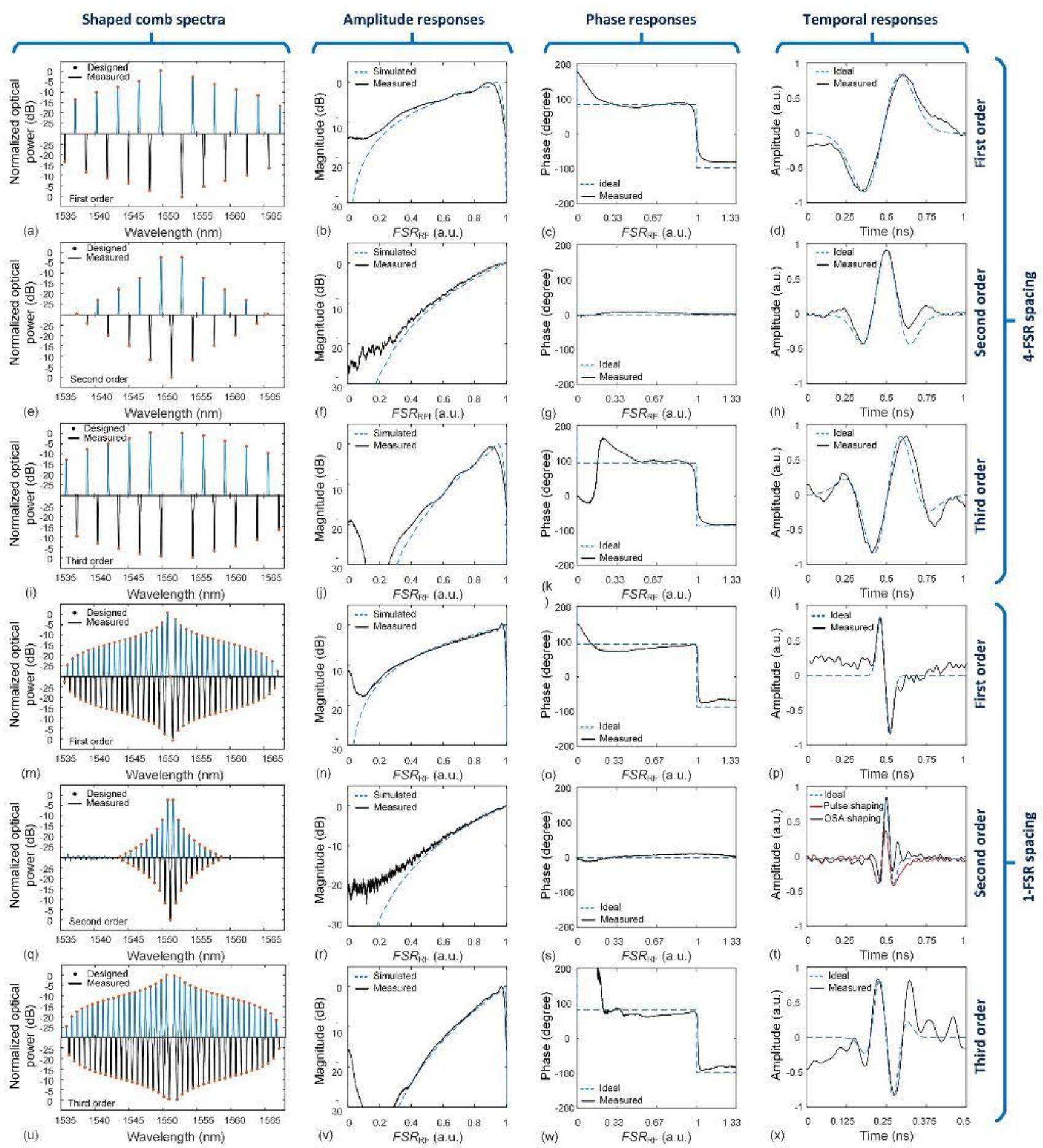

Fig. 10. Simulated and measured first- to third-order differentiators with different comb spacing (single-FSR and 4-FSR). (a) (e) (i) (m) (q) (u) Shaped optical spectral. (b) (f) (j) (n) (r) (v) Amplitude responses. (c) (g) (k) (o) (s) (w) Phase responses. (d) (h) (l) (p) (t) (x) Temporal responses measured with a Gaussian pulse input.

RMSE of time-domain shown in Table 1 has significantly improved.

Also note that the greater number of lines supplied by the soliton crystal micro-comb ( 81 for the 1-FSR spacing) yielded significantly better performance in terms of the spanned number of octaves in the RF domain as well as the RMSE, etc.

On the other hand, the 1-FSR spacing is more limited in operational bandwidth, being restricted to roughly the Nyquist zone of $25 \mathrm{GHz}$. The 2-FSR spacing and 4-FSR spacing system can reach RF frequencies well beyond what conventional electronic microwave technology can achieve. 
Therefore our shaping method gives the flexibility for us to achieve the required system.

\section{Conclusion}

We demonstrate record performance and versatility for soliton crystal micro-comb-based RF signal processing functions by varying wavelength spacing and employing different tap designs and shaping methods. The experimental results agree well with theory, verifying that our soliton crystal micro-comb-based signal processor is a competitive approach towards achieving RF signal processor with broad operation bandwidth, high reconfigurebility, and potentially reduced cost and footprint.

Competing interests: The authors declare no competing interests.

\section{References}

[1] J. Capmany, and D. Novak, "Microwave photonics combines two worlds," Nat. Photonics 1, 319-330 (2007).

[2] J. P. Yao, "Microwave photonics," Journal of Lightwave Technol. 27, (3) 314-335 (2009).

[3] D. Marpaung, J. Yao, and J. Capmany, "Integrated microwave photonics," Nature Photonics 13, 80-90 (2019).

[4] J. Azaña, "Ultrafast analog all-optical signal processors based on fiber-grating devices," IEEE Photonics Journal 2, (3) 359386 (2010).

[5] J. Capmany, B. Ortega, and D. Pastor, "A tutorial on microwave photonic filters," Journal of Lightwave Technol. 24, (1) 201-229 (2006).

[6] V. R. Supradeepa et al., "Comb-based radiofrequency photonic filters with rapid tunability and high selectivity," Nat. Photonics 6, 186-194 (2012).

[7] A. Malacarne et al.,"Single-shot photonic time-intensity integration based on a time-spectrum convolution system," Optics Letters 37, (8) 1355-1357 (2012).

[8] V. Torres-Company, and A. M. Weiner, "Optical frequency comb technology for ultra-broadband radio-frequency photonics," Laser Photonics Rev 8, (3) 368-393 (2014).

[9] Z. Jiang, C. B. Huang, D. E. Leaird, and A. M. Weiner, "Optical arbitrary waveform processing of more than 100 spectral comb lines," Nat Photonics 1, 463-467 (2007).

[10] R. A. Minasian, E. H. W. Chan, and X. Yi, "Microwave photonic signal processing," Optics Express 21, (19) 2291822936 (2013).

[11] J. C. Bancroft, and H. D. Geiger, "Analysis and design of filters for differentiation," CREWES Research Report 9, 21-13 (1997).

[12] Y. Liu, Y. Yu, S. X. Yuan, X. B. Xu, and X. L. Zhang, "Tunable megahertz bandwidth microwave photonic notch filter based on a silica microsphere cavity," Opt. Lett. 41, (21) 50785081 (2016).

[13] D. Marpaung, B. Morrison, M. Pagani, R. Pant, D. Y. Choi, B. Luther-Davies, S. J. Madden, and B. J. Eggleton, "Lowpower, chip-based stimulated Brillouin scattering microwave photonic filter with ultrahigh selectivity," Optica 2, (2) 76-83 (2015).

[14] A. Choudhary, B. Morrison, I. Aryanfar, S. Shahnia, M. Pagani, Y. Liu, K. Vu, S. Madden, D. Marpaung, and B. J. Eggleton, "Advanced integrated microwave signal processing with giant on-chip Brillouin gain," J. Lightwave Technol. 35, (4) 846-854 (2017)

[15] X. Xu et al., "Reconfigurable broadband microwave photonic intensity differentiator based on an integrated optical frequency comb source," APL Photonics 2, (9) 096104 (2017).

[16] X. Q. Zhu, F. Y. Chen, H. F. Peng, and Z. Y. Chen, "Novel programmable microwave photonic filter with arbitrary filtering shape and linear phase," Opt. Express 25, (8) 9232-9243 (2017).

[17] F. Jiang, Y. Yu, H. T. Tang, L. Xu, and X. L. Zhang, "Tunable bandpass microwave photonic filter with ultrahigh stopband attenuation and skirt selectivity," Opt. Express $\mathbf{2 4}$. (16) 18655-18663 (2016).

[18] J. Wu et al., "RF photonics: An optical microcombs' perspective," IEEE J. Sel. Top. Quantum Electron. 24, (4) 6101020 (2018).

[19] E. Hamidi, D. E. Leaird, and A. M. Weiner, "Tunable Programmable Microwave Photonic Filters Based on an Optical Frequency Comb," IEEE Journal of Microwave Theory 58, (11) 3269-3278 (2010).

[20] Z. J. Zhu, H. Chi, T. Jin, S. L. Zheng, X. F. Jin, and X. M. Zhang, "All-positive-coefficient microwave photonic filter with rectangular response," Opt. Lett. 42, (15) 3012-3015 (2017).

[21] S. Mansoori, and A. Mitchell, "RF transversal filter using an AOTF," IEEE Photonic Tech L 16, (3) 879-881 (2004).

[22] J. S. Leng, W. Zhang, and J. A. R. Williams, "Optimization of superstructured fiber Bragg gratings for microwave photonic filters response," IEEE Photonic Tech L 16, (7) 1736-1738 (2004).

[23] M. Delgado-Pinar, J. Mora, A. Diez, M. V. Andres, B. Ortega, and J. Capmany, "Tunable and reconfigurable microwave filter by use of a Bragg-grating-based acousto-optic superlattice modulator," Opt. Lett. 30, (1) 8-10 (2005).

[24] G. Yu, W. Zhang, and J. A. R. Williams, "High-performance microwave transversal filter using fiber Bragg grating arrays," IEEE Photonic Tech L 12, (9) 1183-1185 (2000).

[25] D. B. Hunter, R. A. Minasian, and P. A. Krug, "Tunable optical transversal filter based on chirped gratings," Electron. Lett. 31, (25) 2205-2207 (1995).

[26] X. Xu, M. Tan, J. Wu, A. Boes, B. Corcoran, T. G. Nguyen, S. T. Chu, B. E. Little, R. Morandotti, A. Mitchell, and D. J. Moss, "Photonic RF phase-encoded signal generation with a microcomb source," Journal of Lightwave Technology 38, (7) 1722-727 (2020). DOI:10.1109/JLT.2019.2958564.

[27] M. Tan, X. Xu, B. Corcoran, J. Wu, A. Boes, T. G. Nguyen, S. T. Chu, B. E. Little, R. Morandotti, A. Mitchell, and D. J. Moss, "Microwave and RF photonic fractional Hilbert transformer based on a 50GHz Kerr micro-comb", Journal of Lightwave Technology 37, (24) 6097-6104 (2019).

[28] X. Xu, J. Wu, M. Tan, T. G. Nguyen, S. T. Chu, B. E. Little, R. Morandotti, A. Mitchell, and D. J. Moss, "Broadband microwave frequency conversion based on an integrated optical micro-comb source", Journal of Lightwave Technology 38, (2) 332-338 (2020) 
[29] X. Xu, M. Tan, J. Wu, R. Morandotti, A. Mitchell, and D. J. Moss, "Microcomb-based photonic RF signal processing", IEEE Photonics Technology Letters 31, (23) 1854-1857 (2019).

[30] X. Xu et al., "Photonic microwave true time delays for phased array antennas using a $49 \mathrm{GHz}$ FSR integrated optical microcomb source," Photonics Res. 6, (5) B30-B36 (2018).

[31] X. Xue et al., "Microcomb-based true-time-delay network for microwave beamforming with arbitrary beam pattern control," Journal of Lightwave Technology 36, (12) 2312-2321 (2018).

[32] X. Xu et al., "Advanced RF and microwave functions based on an integrated optical frequency comb source," Opt. Express 26, (3) 2569-2583 (2018).

[33] M. Tan, X. Xu, J. Wu, R. Morandotti, A. Mitchell, and D. J. Moss, "Photonic RF and microwave filters based on $49 \mathrm{GHz}$ and 200GHz Kerr microcombs", Optics Communications 465, Article: 125563, Feb. 22 (2020). doi:10.1016/j.optcom.2020.125563.

[34] X. Xue, et al., "Programmable single-bandpass photonic RF filter based on a Kerr comb from a microring," Journal of Lightwave Technol. 32, (20) 3557-3565 (2014).

[35] X. Xu et al., "Advanced adaptive photonic RF filters with 80 taps based on an integrated optical micro-comb source," Journal of Lightwave Technology 37, (4) 1288-1295 (2019).

[36] T. G. Nguyen et al., "Integrated frequency comb source-based Hilbert transformer for wideband microwave photonic phase analysis," Opt. Express 23, (17) 22087-22097 (2015).

[37] X. Xu et al., "Broadband RF channelizer based on an integrated optical frequency Kerr comb source," Journal of Lightwave Technology 36, (19) 4519-4526 (2018).

[38] X. Xu et al., "High performance RF filters via bandwidth scaling with Kerr micro-combs," APL Photonics 4, 026102 (2019).

[39] X. Xu et al., "Continuously tunable orthogonally polarized RF optical single sideband generator based on micro-ring resonators," Journal of Optics 20, (11) 115701 (2018).

[40] X. Xu et al., "Orthogonally polarized RF optical single sideband generation and dual-channel equalization based on an integrated microring resonator," Journal of Lightwave Technology 36, (20) 4808-4818 (2018).

[41] M. Tan, X. Xu, B. Corcoran, J. Wu, A. Boes, T. G. Nguyen, S. T. Chu, B. E. Little, R. Morandotti, A. Mitchell, and D. J. Moss, "RF and microwave fractional differentiator based on photonics", IEEE Transactions on Circuits and Systems II: Express Briefs, 37 (11) pp. 2767-2771 (2020). DOI: 10.1109/TCSII.2020.2965158.

[42] X. Xu, M. Tan, J. Wu, A. Boes, B. Corcoran, T. G. Nguyen, S. T. Chu, B. E. Little, R. Morandotti, A. Mitchell, and D. J. Moss, "Photonic RF and microwave integrator with soliton crystal microcombs", IEEE Transactions on Circuits and Systems: Express Briefs, 67 (12) pp. 3582-3586 May 19 (2020). DOI:10.1109/TCSII.2020.2995682.

[43] M. Tan, X. Xu, A. Boes, B. Corcoran, J. Wu, T. G. Nguyen, S. T. Chu, B. E. Little, R. Morandotti, A. Mitchell, D. J. Moss, "Photonic RF arbitrary waveform generator based on a soliton crystal micro-comb source", Journal of Lightwave Technology, 38 (22) pp.6221-6226, Oct 22 (2020).

DOI: 10.1109/JLT.2020.3009655.

[44] X. Xu, M. Tan, J. Wu, A. Boes, T. G. Nguyen, S. T. Chu, B. E. Little, R. Morandotti, A. Mitchell, and D. J. Moss,
"Broadband photonic radio frequency channelizer with 90 channels based on a soliton crystal microcomb", Journal of Lightwave Technology, 38, (18) pp. 5116 - 5121, Sept. 15 (2020). doi: 10.1109/JLT.2020.2997699.

[45] A. J. Metcalf et al., "Integrated line-by-line optical pulse shaper for high-fidelity and rapidly reconfigurable RFfiltering," Optics Express 24, (21) 23925-23940 (2016).

[46] J. McClellan, T. W. Parks, and L. Rabiner, "A computer program for designing optimum FIR linear phase digital filters," Transactions on Audio and Electroacoustics 21, (6) 506-526 (1973).

[47] R. Wu, V. R. Supradeepa, C. M. Long, D. E. Leaird, and A. M. Weiner, "Generation of very flat optical frequency combs from continuous-wave lasers using cascaded intensity and phase modulators driven by tailored radio frequency waveforms," Opt. Lett. 35, (19) 3234-3236 (2010).

[48] W. Z. Li, and J. P. Yao, "Optical frequency comb generation based on repeated frequency shifting using two Mach-Zehnder modulators and an asymmetric Mach-Zehnder interferometer," Opt. Express 17, (26) 23712-23718 (2009).

[49] C. H. Chen, C. He, D. Zhu, R. H. Guo, F. Z. Zhang, and S. L. Pan, "Generation of a flat optical frequency comb based on a cascaded polarization modulator and phase modulator," Opt. Lett. 38, (16) 3137-3139 (2013).

[50] T. Saitoh, M. Kourogi, and M. Ohtsu, "An optical frequency synthesizer using a waveguide-type optical frequency comb generator at 1.5-mu m wavelength," IEEE Photonic Tech L 8, (11) 1543-1545 (1996).

[51] P. del'Haye et al., "Optical frequency comb generation from a monolithic Micro-resonator," Nature 450, 1214-1217 (2007).

[52] W. Liang et al., "High spectral purity Kerr frequency comb radio frequency photonic oscillator," Nature communications $\mathbf{6}$, 7957 (2015).

[53] J. S. Levy et al., "CMOS-compatible multiple-wavelength oscillator for on-chip optical interconnects," Nat. Photonics 4, 37-40 (2010).

[54] L. Razzari et al., "CMOS-compatible integrated optical hyper-parametric oscillator," Nat. Photonics 4, 41-45 (2010).

[55] D. J. Moss, R. Morandotti, A. L. Gaeta, and M. Lipson, "New CMOS-compatible platforms based on silicon nitride and Hydex for nonlinear optics," Nat. Photonics 7, 597-607 (2013).

[56] T. J. Kippenberg, A. L. Gaeta, M. Lipson, and M. L. Gorodetsky, "Dissipative Kerr solitons in optical microresonators," Science 361, eaan8083 (2018).

[57] A. L. Gaeta, M. Lipson, and T. J. Kippenberg, "Photonicchip-based frequency combs," Nature Photonics 13, 158-169 (2019).

[58] A. Pasquazi et al., "Micro-combs: A novel generation of optical sources," Physics Reports-Review Section of Physics Letters 729, 1-81 (2018).

[59] D. C. Cole, E. S. Lamb, P. Del'Haye, S. A. Diddams, and S. B. Papp, "Soliton crystals in Kerr resonators," Nat. Photonics 11, 671-676 (2017).

[60] X. Xue et al., "Mode-locked dark pulse Kerr combs in normal-dispersion microresonators," Nature Photonics 9, 594 (2015).

[61] H. Bao et al., "Laser cavity-soliton microcombs," Nature Photonics 13, 384-389 (2019). 
[62] X. Xue, X. Zheng, and B. Zhou, "Super-efficient temporal solitons in mutually coupled optical cavities," Nature Photonics 13, 616-622 (2019).

[63] H. Zhou et al., "Soliton bursts and deterministic dissipative Kerr soliton generation in auxiliary-assisted microcavities," Light: Science \& Applications 8, 50 (2019).

[64] B. Stern, X Ji, Y. Okawachi, A.L. Gaeta, M. Lipson, "Batteryoperated integrated frequency comb generator," Nature 562, 401-405 (2018).

[65] M. Ferrera, L. Razzari, D. Duchesne, R. Morandotti, Z. Yang, M. Liscidini, J. E. Sipe, S. Chu, B. E. Little, and D. J. Moss, "Low-power continuous-wave nonlinear optics in doped silica glass integrated waveguide structures," Nature Photonics $\mathbf{2}$, 737-740 (2008).

[66] Peccianti, A. Pasquazi, Y. Park, B. E. Little, S. T. Chu, D. J. Moss, and R. Morandotti, "Demonstration of a stable ultrafast laser based on a nonlinear microcavity," Nat. Commun. 3, 1-6 (2012).

[67] A. Pasquazi, M. Peccianti, B. E. Little, S. T. Chu, D. J. Moss, and R. Morandotti, "Stable, dual mode, high repetition rate mode-locked laser based on a microring resonator," Opt. Exp. 20, (24) 27355-27362 (2012).

[68] A. Pasquazi, L. Caspani, M. Peccianti, M. Clerici, M. Ferrera, L. Razzari, D. Duchesne, B. E. Little, S. T. Chu, D. J. Moss, and R. Morandotti, "Self-locked optical parametric oscillation in a CMOS compatible microring resonator: a route to robust optical frequency comb generation on a chip," Opt. Exp. 21, (11) 13333-13341 (2013).

[69] A. Pasquzai, Y. Park, J. Azana, F. Legare, R. Morandotti, B. E. Little, S. T. Chu, and D. J. Moss, "Efficient wavelength conversion and net parametric gain via Four wave Mixing in a high index doped silica waveguide," Opt. Exp. 18, (8) 76347641 (2010).

[70] M. Peccianti, M. Ferrera, L. Razzari, R. Morandotti, B. E. Little, S. T. Chu, and D. J. Moss, "Subpicosecond optical pulse compression via an integrated nonlinear chirper," Opt. Exp. 18, (8) $7625-7633$ (2010).

[71] D. Duchesne, M. Peccianti, M. R. E. Lamont, M. Ferrera, L. Razzari, F. Legare, R. Morandotti, S. Chu, B. E. Little, and D. J. Moss, "Supercontinuum generation in a high index doped silica glass spiral waveguide," Opt. Exp. 18, (2) 923-930 (2010).

[72] H. Bao, L. Olivieri, M. Rowley, S. T. Chu, B. E. Little, R. Morandotti, D. J. Moss, J. S. T. Gongora, M. Peccianti and A. Pasquazi, "Turing patterns in a fibre laser with a nested microresonator: robust and controllable micro-comb generation", Physical Review Research 2, 023395 (2020).

[73] L. D. Lauro, J. Li, D. J. Moss, R. Morandotti, S. T. Chu, M. Peccianti, and A. Pasquazi, "Parametric control of thermal selfpulsation in micro-cavities," Opt. Lett. 42, (17) 3407-3410 (2017).

[74] H. Bao, A. Cooper, S. T. Chu, D. J. Moss, R. Morandotti, B. E. Little, M. Peccianti, and A. Pasquazi, "Type-II micro-comb generation in a filter-driven four wave mixing laser," Photonics Research 6, (5) B67-B73 (2018).

[75] M. Ferrera, Y. Park, L. Razzari, B. E. Little, S. T. Chu, R. Morandotti, D. J. Moss, and J. Azaña, "On-Chip ultra-fast 1st and 2nd order CMOS compatible all-optical integration", Optics Express 19, (23) 23153-23161 (2011).
[76] W. Wang, Z. Lu, W. Zhang, S. T. Chu, B. E. Little, L. Wang, X. Xie, M. Liu, Q. Yang and L. Wang, "Robust soliton crystals in a thermally controlled microresonator," Optics Letters 43, (9) 2002-2005 (2018).

[77] B. Corcoran, M. Tan, X. Xu, A. Boes, J. Wu, T. G. Nguyen, S. T. Chu, B. E. Little, R. Morandotti, A. Mitchell, D. J. Moss, "Ultra-dense optical data transmission over standard fibre with a signal chip source," Nature Communications 11, 1-7, Article 2568 (2020).

[78] P. Marin-Palomo, el al., "Microresonator-based solitons for massively parallel coherent optical communication", Nature 546 (7657), 274 (2017).

[79] J. Pfeifle, V. Brasch, M. Lauermann, Y. Yu, D. Wegner, T. Herr, K. Hartinger, et al., "Coherent terabit communications with microresonator Kerr frequency combs", Nature Photonics 8 (5), 375-380 (2014).

[80] X. Xu, et al., "Photonic perceptron based on a Kerr microcomb for scalable high speed optical neural networks", Laser and Photonics Reviews 14 (10), 2000070 (2020).

[81] X. Xu, et al., "11 TOPS photonic convolutional accelerator for optical neural networks," Nature $\mathbf{5 8 9}$ (7840), 44-51 (2021).

[82] J. Feldmann, et al., "Parallel convolutional processing using an integrated photonic tensor core," Nature $\mathbf{5 8 9}$ (7840), 52-58 (2021).

[83] Kues, M. et al. Quantum optical microcombs. Nature Photonics 13, (3) 170-179 (2019). doi:10.1038/s41566-0190363-0

[84] C. Reimer et al., "Integrated frequency comb source of heralded single photons," Optics Express, vol. 22, no. 6, pp. 6535-6546, 2014.

[85] C. Reimer, et al., "Cross-polarized photon-pair generation and bi-chromatically pumped optical parametric oscillation on a chip", Nature Communications, vol. 6, Article 8236, 2015. DOI: $10.1038 /$ ncomms 9236 .

[86] L. Caspani, C. Reimer, M. Kues, et al., "Multifrequency sources of quantum correlated photon pairs on-chip: a path toward integrated Quantum Frequency Combs," Nanophotonics, vol. 5, no. 2, pp. 351-362, 2016.

[87] C. Reimer et al., "Generation of multiphoton entangled quantum states by means of integrated frequency combs," Science, vol. 351, no. 6278, pp. 1176-1180, 2016.

[88] M. Kues, et al., "On-chip generation of high-dimensional entangled quantum states and their coherent control", Nature, vol. 546, no. 7660, pp. 622-626, 2017.

[89] P. Roztocki et al., "Practical system for the generation of pulsed quantum frequency combs," Optics Express, vol. 25, no.16, 18940-18949, 2017.

[90] Y. Zhang, et al., "Induced photon correlations through superposition of two four-wave mixing processes in integrated cavities", Laser and Photonics Reviews, vol. 14, no. 7, pp. 2000128, 2020. DOI: 10.1002/lpor.202000128

[91] C. Reimer, et al.,"High-dimensional one-way quantum processing implemented on d-level cluster states", Nature Physics, vol. 15 (2) 148 (2019).

[92] P. Roztocki et al., "Arbitrary phase access for stable fiber interferometers", Laser and Photonics Reviews, vol.15 (4) 2000524 (2021). DOI: 10.1002/lpor.202000524. 
[93] S. Sciara et al., "Generation and Processing of Complex Photon States With Quantum Frequency Combs", IEEE Photonics Technology Letters, vol. 31 (23), 1862-1865 (2019).

[94] P. Roztocki et al., "Complex quantum state generation and coherent control based on integrated frequency combs", Journal of Lightwave Technology, vol. 37 (2), 338-344 (2019).

[95] S. Sciara et al., "Universal N-Partite d-Level Pure-State Entanglement Witness Based on Realistic Measurement Settings", Physical Review Letters, Vol. 122 (12), 120501 (2019).

[96] L.Jin, L. Di Lauro, A.Pasquazi, M. Peccianti, David J. Moss, R.Morandotti, B.E. Little, S.T.Chu, "Optical multi-stability in a nonlinear high-order microring resonator filter", Editors Pick, Applied Physics Letters (APL) Photonics, vol. $\underline{5}$ Article 056106, May 22 (2020). https://doi.org/10.1063/5.0002941

[97] A. Aadhi, Anton V. Kovalev, Michael Kues, Piotr Roztocki, Christian Reimer, Young Zhang, Tao Wang, Brent E. Little, Sai T. Chu, Zhiming Wang, David J. Moss, Evgeny A. Viktorov, and Roberto Morandotti, "Highly reconfigurable hybrid laser based on an integrated nonlinear waveguide", Optics Express, vol. 27 (18) 25252 (2019). DOI: 10.1364/OE.27.025251.

[98] M. Karpov, M. H. P. Pfeiffer, H Guo, W. Weng, J. Liu, T. J. Kippenberg, "Dynamics of soliton crystals in optical microresonators", Nature Physics 15 (10), 1071-1077 (2019).

[99] M. Tan, X. Xu, J. Wu, R. Morandotti, A. Mitchell, and D. J. Moss, "RF and microwave high bandwidth signal processing based on Kerr Micro-combs", Advances in Physics X, VOL. 6, NO. 1, 1838946 (2020). DOI:10.1080/23746149.2020.1838946.

[100] Mengxi Tan, Xingyuan Xu, Jiayang Wu, Thach G. Nguyen, Sai T. Chu, Brent E. Little, Roberto Morandotti, Arnan Mitchell, and David J. Moss, "Photonic Radio Frequency Channelizers based on Kerr Micro-combs and Integrated Microring Resonators", JOSarXiv.202010.0002.

[101] Mengxi Tan, Xingyuan Xu, David Moss "Tunable Broadband RF Photonic Fractional Hilbert Transformer Based on a Soliton Crystal Microcomb", Preprints, DOI: 10.20944/preprints202104.0162.v1

[102] M. Tan, X. Xu, J. Wu, T. G. Nguyen, S. T. Chu, B. E. Little, R. Morandotti, A. Mitchell, and David J. Moss, "Orthogonally polarized Photonic Radio Frequency single sideband generation with integrated micro-ring resonators", Journal of Semiconductors $\underline{42}$ (4), 041305 (2021). DOI: 10.1088/16744926/42/4/041305.

[103] M. Tan, X. Xu, J. Wu, T. G. Nguyen, S. T. Chu, B. E. Little, R. Morandotti, A. Mitchell, and David J. Moss, "Photonic Radio Frequency Channelizers based on Kerr Optical Microcombs", Journal of Semiconductors $\underline{42}$ (4), 041302 (2021). DOI:10.1088/1674-4926/42/4/041302.

[104] H.Bao, L.Olivieri, M.Rowley, S.T. Chu, B.E. Little, R.Morandotti, D.J. Moss, J.S.T. Gongora, M.Peccianti and A.Pasquazi, "Laser Cavity Solitons and Turing Patterns in Microresonator Filtered Lasers: properties and perspectives", Paper No. LA203-5, Paper No. 11672-5, SPIE LASE, SPIE Photonics West, San Francisco CA March 6-11 (2021). DOI: $10.1117 / 12.2576645$

[105] M. Tan, X. Xu, J. Wu, A. Boes, T. G. Nguyen, S. T. Chu, B. E. Little, R. Morandotti, A. Mitchell, and David J. Moss, "Advanced microwave signal generation and processing withl soliton crystal microcombs", or "Photonic convolutional [119] accelerator and neural network in the Tera-OPs regime based on Kerr microcombs", Paper No. 11689-38, PW21O-OE20167, Integrated Optics: Devices, Materials, and Technologies XXV, SPIE Photonics West, San Francisco CA March 6-11 (2021). DOI: $10.1117 / 12.2584017$

[106] Mengxi Tan, Bill Corcoran, Xingyuan Xu, Andrew Boes, Jiayang Wu, Thach Nguyen, Sai T. Chu, Brent E. Little, Roberto Morandotti, Arnan Mitchell, and David J. Moss, "Optical data transmission at 40 Terabits/s with a Kerr soliton crystal microcomb", Paper No.11713-8, PW21O-OE803-23, Next-Generation Optical Communication: Components, SubSystems, and Systems X, SPIE Photonics West, San Francisco CA March 6-11 (2021). DOI:10.1117/12.2584014

[107] M. Tan, X. Xu, J. Wu, A. Boes, T. G. Nguyen, S. T. Chu, B. E. Little, R. Morandotti, A. Mitchell, and David J. Moss, "RF and microwave photonic, fractional differentiation, integration, and Hilbert transforms based on Kerr microcombs", Paper No. 11713-16, PW21O-OE803-24, NextGeneration Optical Communication: Components, SubSystems, and Systems X, SPIE Photonics West, San Francisco CA March 6-11 (2021). DOI:10.1117/12.2584018

[108] M. Tan, X. Xu, J. Wu, A. Boes, T. G. Nguyen, S. T. Chu, B. E. Little, R. Morandotti, A. Mitchell, and David J. Moss, "Broadband photonic RF channelizer with 90 channels based on a soliton crystal microcomb", or "Photonic microwave and RF channelizers based on Kerr micro-combs", Paper No. 11685-22, PW21O-OE106-49, Terahertz, RF, Millimeter, and Submillimeter-Wave Technology and Applications XIV, SPIE Photonics West, San Francisco CA March 6-11 (2021). DOI:10.1117/12.2584015

[109] X. Xu, M. Tan, J. Wu, S. T. Chu, B. E. Little, R. Morandotti, A. Mitchell, B. Corcoran, D. Hicks, and D. J. Moss, "Photonic perceptron based on a Kerr microcomb for scalable high speed optical neural networks", IEEE Topical Meeting on Microwave Photonics (MPW), pp. 220-224,. Matsue, Japan, November 24-26, 2020. Electronic ISBN:978-488552-331-1. DOI: $10.23919 / M W P 48676.2020 .9314409$

[110] Mengxi Tan, Bill Corcoran, Xingyuan Xu, Andrew Boes, Jiayang $\mathrm{Wu}$, Thach Nguyen, S.T. Chu, B. E. Little, Roberto Morandotti, Arnan Mitchell, and David J. Moss, "Ultra-high bandwidth optical data transmission with a microcomb", IEEE Topical Meeting on Microwave Photonics (MPW), pp. 78-82.Virtual Conf., Matsue, Japan, November 24-26, 2020. Electronic ISBN:978-4-88552-331-1. DOI: $10.23919 / M W P 48676.2020 .9314476$

[115] X. Xu et al., "11 TeraFLOPs per second photonic convolutional accelerator for deep learning optical neural networks", arXiv preprint arXiv:2011.07393 (2020).

[116] D. Moss, "11 Tera-FLOP/s photonic convolutional accelerator and deep learning optical neural networks", Research Square (2021). DOI: https://doi.org/10.21203/rs.3.rs-493347/v1.

[117] D. Moss, "11.0 Tera-FLOP/second photonic convolutional accelerator for deep learning optical neural networks", TechRxiv. Preprint

(2020). https://doi.org/10.36227/techrxiv.13238423.v1.

[118] Moss, D., "11 Tera-flop/s Photonic Convolutional Accelerator for Optical Neural Networks." OSF Preprints, 23 Feb. (2021). DOI: $10.31219 /$ osf.io/vqt4s.

M. Tan, X. Xu, J. Wu, T. G. Nguyen, S. T. Chu, B. E. Little, R. Morandotti, A. Mitchell, and David J. Moss, "Photonic Radio 
Frequency Channelizers based on Kerr Optical Micro-combs", Journal of Semiconductors, Vol. 42, No. 4, 041302 (2021). (ISSN 1674-4926). DOI:10.1088/1674-4926/42/4/041302.

[120] M. Tan, X. Xu, J. Wu, D.J. Moss, "High bandwidth temporal RF photonic signal processing with Kerr micro-combs: integration, fractional differentiation and Hilbert transforms", arXiv preprint arXiv:2103.03674 (2021).

[121] D. Moss, "Temporal RF photonic signal processing with Kerr micro-combs: Hilbert transforms, integration and fractional differentiation", OSF Preprints, 18 Feb. (2021). DOI: 10.31219/osf.io/hx9gb.

[122] D. Moss, "RF and microwave photonic high bandwidth signal processing based on Kerr micro-comb sources", TechRxiv. Preprint (2020). DOI:10.36227/techrxiv.12665609.v3.

[123] D. Moss, "RF and microwave photonic signal processing with Kerr micro-combs", Research Square (2021). DOI: 10.21203/rs.3.rs473364/v1

[124] M. Tan, X. Xu, J. Wu, D.J. Moss, "RF Photonic Signal Processing with Kerr Micro-Combs: Integration, Fractional Differentiation and Hilbert Transforms", Preprints (2020). 2020090597. doi:10.20944/preprints202009.0597.v1.

[125] Mengxi Tan, Xingyuan Xu, Jiayang Wu, Thach G. Nguyen, Sai T. Chu, Brent E. Little, Roberto Morandotti, Arnan Mitchell, and David J. Moss, "Photonic Radio Frequency Channelizers based on Kerr Micro-combs and Integrated Micro-ring Resonators", JOSarXiv.202010.0002.

[126] Mengxi Tan, Xingyuan Xu, David Moss "Tunable Broadband RF Photonic Fractional Hilbert Transformer Based on a Soliton Crystal Microcomb", Preprints, DOI: 10.20944/preprints202104.0162.v1

[127] M. Tan, X. Xu, J. Wu, T. G. Nguyen, S. T. Chu, B. E. Little, R. Morandotti, A. Mitchell, and David J. Moss, "Orthogonally polarized Photonic Radio Frequency single sideband generation with integrated micro-ring resonators", Journal of Semiconductors vol. 42, No.4, 041305 (2021). DOI: 10.1088/16744926/42/4/041305.

[128] M. Tan, X. Xu, J. Wu, T. G. Nguyen, S. T. Chu, B. E. Little, R. Morandotti, A. Mitchell, and David J. Moss, "Photonic Radio Frequency Channelizers based on Kerr Optical Micro-combs", Journal of Semiconductors 42 (4), 041302 (2021). (ISSN 16744926). DOI:10.1088/1674-4926/42/4/041302.

[129] Mengxi Tan, Xingyuan Xu, David Moss "Tunable Broadband RF Photonic Fractional Hilbert Transformer Based on a Soliton Crystal Microcomb", Preprints, DOI: 10.20944/preprints202104.0162.v1

[130] M. Tan, X. Xu, J. Wu, T. G. Nguyen, S. T. Chu, B. E. Little, R. Morandotti, A. Mitchell, and David J. Moss, "Orthogonally polarized Photonic Radio Frequency single sideband generation with integrated micro-ring resonators", Journal of Semiconductors 42 (4), 041305 (2021). DOI: 10.1088/1674-4926/42/4/041305.

[131] A. Pasquazi, et al., "Sub-picosecond phase-sensitive optical pulse characterization on a chip", Nature Photonics, vol. 5, no. 10, pp. 618-623 (2011). DOI: 10.1038/nphoton.2011.199.

[132] D. Duchesne, M. Peccianti, M. R. E. Lamont, et al., "Supercontinuum generation in a high index doped silica glass spiral waveguide," Optics Express, vol. 18, no, 2, pp. 923-930 (2010).

[133] M. Ferrera, et al., "On-chip CMOS-compatible all-optical integrator", Nature Communications, vol. 1, Article 29 (2010).

[134] H. Bao et al., "Turing patterns in a fibre laser with a nested microresonator: robust and controllable micro-comb generation", Physical Review Research, vol. 2, pp. 023395 (2020).

[135] L. D. Lauro, J. Li, D. J. Moss, R. Morandotti, S. T. Chu, M52] Peccianti, and A. Pasquazi, "Parametric control of thermal self- pulsation in micro-cavities," Opt. Lett. vol. 42, no. 17, pp. 3407-3410, Aug. 2017.

[136] H. Bao et al., "Type-II micro-comb generation in a filter-driven four wave mixing laser," Photonics Research, vol. 6, no. 5, pp. B67-B73 (2018).

[137] A. Pasquazi, et al., "All-optical wavelength conversion in an integrated ring resonator," Optics Express, vol. 18, no. 4, pp. 3858-3863 (2010).

[138] A. Pasquazi, Y. Park, J. Azana, et al., "Efficient wavelength conversion and net parametric gain via Four Wave Mixing in a high index doped silica waveguide," Optics Express, vol. 18, no. 8, pp. 7634-7641 (2010).

[139] M. Peccianti, M. Ferrera, L. Razzari, et al., "Subpicosecond optical pulse compression via an integrated nonlinear chirper," Optics Express, vol. 18, no. 8, pp. 7625-7633 (2010).

[140] D. Duchesne, M. Ferrera, L. Razzari, et al., "Efficient selfphase modulation in low loss, high index doped silica glass integrated waveguides," Optics Express, vol. 17, no. 3, pp. 1865-1870 (2009).

[141] M. Peccianti, et al., "Demonstration of an ultrafast nonlinear microcavity modelocked laser", Nature Communications, vol. 3, pp. 765 (2012).

[142] M. Kues, et al., "Passively modelocked laser with an ultranarrow spectral width", Nature Photonics, vol. 11, no. 3, pp. 159 (2017). DOI:10.1038/nphoton.2016.271

[143] A. Pasquazi, L. Caspani, M. Peccianti, et al., "Self-locked optical parametric oscillation in a CMOS compatible microring resonator: a route to robust optical frequency comb generation on a chip," Optics Express, vol. 21, no. 11, pp. 13333-13341 (2013).

[144] A. Pasquazi, M. Peccianti, B. E. Little, et al., "Stable, dual mode, high repetition rate mode-locked laser based on a microring resonator," Optics Express, vol. 20, no. 24, pp. 27355-27362 (2012)

[145] C. Reimer, L. Caspani, M. Clerici, et al., "Integrated frequency comb source of heralded single photons," Optics Express, vol. 22, no. 6, pp. 6535-6546 (2014).

[146] L. Caspani, C. Reimer, M. Kues, et al., "Multifrequency sources of quantum correlated photon pairs on-chip: a path toward integrated Quantum Frequency Combs," Nanophotonics, vol. 5, no. 2, pp. 351-362 (2016).

[147] C. Reimer, M. Kues, P. Roztocki, B. Wetzel, F. Grazioso, B. E. Little, S. T. Chu, T. Johnston, Y. Bromberg, L. Caspani, D. J. Moss, and R. Morandotti, "Generation of multiphoton entangled quantum states by means of integrated frequency combs," Science, vol. 351, no. 6278, pp. 1176-1180 (2016).

[148] Y. Zhang, et al., "Induced photon correlations through superposition of two four-wave mixing processes in integrated cavities", Laser and Photonics Reviews, vol. 14, no. 7, 2000128 (2020). DOI: 10.1002/lpor.202000128

[149] M. Kues, C. Reimer, A. Weiner, J. Lukens, W. Munro, D. J. Moss, and R. Morandotti, "Quantum Optical Micro-combs", Nature Photonics, vol. 13, no.3, pp. 170-179 (2019).

[150] C. Reimer, et al., "High-dimensional one-way quantum processing implemented on d-level cluster states", Nature Physics, vol. 15, no.2, pp. 148-153 (2019).

[151] T.Ido, H.Sano, D.J.Moss, S.Tanaka, and A.Takai, "Strained InGaAs/InAlAs MQW electroabsorption modulators with large bandwidth and low driving voltage", Photonics Technology Letters, Vol. 6, 1207 (1994). DOI: 10.1109/68.329640.

H. Arianfard et al., "Three waveguide coupled sagnac loop reflectors for advanced spectral engineering," J. Lightwave Technol., doi: 10.1109/JLT.2021.3066256. 
[153] J. Wu et al., "Nested configuration of silicon microring resonator with multiple coupling regimes," IEEE Photon. Technol. Lett., vol. 25, no. 6, pp. 580-583, Mar. 2013.

[154] J. Wu, T. Moein, X. Xu, and D. J. Moss, “Advanced photonic filters based on cascaded Sagnac loop reflector resonators in silicon-oninsulator nanowires," APL Photonics, vol. 3, 046102 (2018). DOI:/10.1063/1.5025833Apr. 2018.

[155] J. Wu, T. Moein, X. Xu, D.J. Moss, "Silicon photonic filters based on cascaded Sagnac loop resonators", arXiv preprint arXiv:1805.05405a (2018).

[156] J. Wu, T. Moein, X. Xu, G. H. Ren, A. Mitchell, and D. J. Moss, "Micro-ring resonator quality factor enhancement via an integrated Fabry-Perot cavity," APL Photonics, vol. 2, 056103 (2017).

[157] H. Arianfard, J. Wu, S. Juodkazis, and D. J. Moss, "Advanced Multi-Functional Integrated Photonic Filters Based on Coupled Sagnac Loop Reflectors", Journal of Lightwave Technology, Vol. 39, No.5, pp.1400-1408 (2021). DOI: 10.1109/JLT.2020.3037559.

[158] D. J. Moss, "Optimization of Optical Filters based on Integrated Coupled Sagnac Loop Reflectors", Research Square (2021). DOI: https://doi.org/10.21203/rs.3.rs-478204/v1

[159] H. Arianfard, J. Wu, S. Juodkazis, D. J. Moss, "Spectral Shaping Based on Integrated Coupled Sagnac Loop Reflectors Formed by a Self-Coupled Wire Waveguide", submitted, IEEE Photonics Technology Letters, vol. 33 (2021).

[160] J. Wu et al., "Graphene oxide waveguide and micro-ring resonator polarizers," Laser Photonics Rev., vol. 13, no. 9, pp. 1900056, Aug. 2019.

[161] Y. Zhang et al., "Optimizing the Kerr nonlinear optical performance of silicon waveguides integrated with 2D graphene oxide films," J. Lightwave Technol., doi: 10.1109/JLT.2021.3069733.

[162] Y. Qu et al., "Analysis of four-wave mixing in silicon nitride waveguides integrated with 2D layered graphene oxide films," J. Lightwave Technol., vol. 39, no. 9, pp. 2902-2910, May. 2021.

[163] Y. Qu et al., "Enhanced four-wave mixing in silicon nitride waveguides integrated with 2D layered graphene oxide films," Adv. Opt. Mater., vol. 8, no. 20, pp. 2001048, Oct. 2020.

[164] J. Wu et al., "2D layered graphene oxide films integrated with micro-ring resonators for enhanced nonlinear optics," Small, vol. 16, no. 16, pp. 1906563, Mar. 2020.

[165] Moss, David; Wu, Jiayang; Xu, xingyuan; Yang, Yunyi; Jia, Linnan; Zhang, Yuning; et al. (2020): Enhanced optical four-wavemixing in integrated ring resonators with graphene oxide films. TechRxiv. Preprint. DOI:10.36227/techrxiv.11859429.v1.

[166] Wu, J.; Yang, Y.; Qu, Y.; Jia, L.; Zhang, Y.; Xu, X.; Chu, S.T.; Little, B.E.; Morandotti, R.; Jia, B.; Moss, D.J. Enhancing Third Order Nonlinear Optics in Integrated Ring Resonators with 2D Material Films. Preprints 2020, 2020030107

[167] Wu, J.; Yang, Y.; Qu, Y.; Jia, L.; Zhang, Y.; Xu, X.; Chu, S.T.; Little, B.E.; Morandotti, R.; Jia, B.; Moss, D.J. Enhancing Third Order Nonlinear Optics in Integrated Ring Resonators with 2D Material Films. Preprints 2020, 2020030107

[168] J. Wu et al., "Enhanced four-wave-mixing with 2D layered graphene oxide films integrated with CMOS compatible micro-ring resonators", arXiv preprint, arXiv:2002.04158 (2020).

[169] Y. Zhang et al., "Enhanced Kerr nonlinearity and nonlinear figure of merit in silicon nanowires integrated with 2D graphene oxide films," ACS Appl. Mater. Interfaces, vol. 12, no. 29, pp. $33094-$ 33103, Jun. (2020).।

[170] Y. Zhang et al., "Enhanced nonlinear optical figure-of-merit at $1550 \mathrm{~nm}$ for silicon nanowires integrated with graphene oxide layered films", arXiv preprint arXiv:2004.08043 (2020).

[171] D. Moss, "Elevating silicon into a high performance nonlinear optical platform through the integration of $2 \mathrm{D}$ graphene oxide thin films", Research Square (2021). DOI: https://doi.org/10.21203/rs.3.rs-511259/v1.

[172] Moss, D.; Wu, J.; Jia, B.; Yang, Y.; Qu, Y.; Jia, L.; Zhang, Y. "Improved Nonlinear Optics in Silicon-on-insulator Nanowires Integrated with 2D Graphene Oxide Films", Preprints (2020), 2020040033.

[173] Y. Yang et al., "Invited article: enhanced four-wave mixing in waveguides integrated with graphene oxide," APL Photonics, vol. 3, no. 12, pp. 120803, Oct. 2018.

[174] T.D. Vo et al., "Silicon-chip-based real-time dispersion monitoring for $640 \mathrm{Gbit} / \mathrm{s}$ DPSK signals", Journal of Lightwave Technology, vol. 29, no. 12, 1790-1796 (2011). 